\title{
Aplicación de vermicomposta y producción de biomasa en cultivo de maíz de temporal
}

\author{
Application of vermicompost and biomass production in temporary maize crop
}

\author{
González-Cortés J. C. ${ }^{1 凶}$, Ramírez-Mandujano C. A. ${ }^{1}$, Avila-Bautista A. ${ }^{1}$ \\ ${ }^{1}$ Facultad de biología-Universidad Michoacana de San Nicolás de Hidalgo, Calle Francisco J. Mujica s/n, \\ Edificio R Ciudad Universitaria, Morelia, Michoacán (014433267115) \\ ${ }^{\square}$ Autor para correspondencia: jcgonzalezcortes@yahoo.com.mx
}

Recibido: $15 / 07 / 2017$

Aceptado: 10/12/2017

\section{RESUMEN}

La pérdida de fertilidad en suelos agrícolas es un problema mundial, donde las prácticas de manejo son determinantes, como el excesivo uso de agroquímicos y mínima reincorporación de materia orgánica. Ante esta problemática el uso de materiales orgánicos es una práctica que debe retomarse si se desea lograr sistemas productivos sostenibles. La vermicomposta se ha venido probando en los últimos años, considerando el beneficio físico, químico y biológico al suelo. El maíz de temporal es producido en Michoacán en condiciones de baja o nula rentabilidad, por el elevado costo de los fertilizantes químicos. El objetivo del presente trabajo fue evaluar diferentes dosis de vermicomposta combinada con dosis reducidas de fertilizante químico (50\%) sobre la producción de biomasa vegetal y de grano, en maíz de temporal. Se probaron en campo cuatro dosis de vermicomposta: 4, 8, 16 y $32 \mathrm{t} \mathrm{ha}^{-1}$, con un testigo absoluto y un tratamiento químico convencional. Los resultados mostraron un efecto positivo de la vermicomposta sobre el desarrollo vegetativo, en la producción de biomasa de hojas, tallos y raíces, así como en la biomasa del grano (Tukey $\mathrm{p}<0.05$ ), superando siempre al testigo absoluto y compitiendo con el tratamiento químico. Los tratamientos con 16 y $32 \mathrm{t} \mathrm{ha}^{-1}$ tuvieron las medias de rendimiento más altas $\left(4.55\right.$ y $\left.4.46 \mathrm{t} \mathrm{ha}^{-1}\right)$. En las variables: número de granos, número de hileras y porcentaje de humedad del grano no se obtuvieron diferencias significativas. Es posible la reducción gradual de fertilizantes químicos utilizando vermicomposta sin afectar la producción de grano.

Palabras clave: Biomasa, Fertilidad, Maíz, Sostenible, Vermicomposta.

\begin{abstract}
ABSTRAC
Loss of fertility in agricultural soils is a global problem, where management practices are determinant, such as excessive use of agrochemicals and minimal reincorporation of organic matter. Faced with this problem, the use of organic materials is a practice that must be resumed if sustainable production systems are to be achieved. The vermicomposta has been tried in the last years, considering the physical, chemical and biological benefit to the soil. Temporary maize is produced in Michoacán under conditions of low
\end{abstract}


or zero profitability, by the high cost of chemical fertilizers. The objective of the present work was to evaluate different doses of vermicompost combined with reduced doses of chemical fertilizer $(50 \%)$ on the production of plant and grain biomass in temporary maize. Four doses of vermicompost were tested in the field: 4, 8, 16 and $32 \mathrm{t} \mathrm{ha}^{-1}$, with an absolute control and conventional chemical treatment. The results showed a positive effect of the vermicompost on the vegetative development, on biomass of leaves, stems and roots, as well as on the grain biomass (Tukey $\mathrm{p}<0.05$ ), always surpassing the absolute control and competing with the chemical treatment. Treatments with 16 and $32 \mathrm{t} \mathrm{ha}^{-1}$ had the highest yield means $\left(4.55\right.$ y $\left.4.46 \mathrm{t} \mathrm{ha}^{-1}\right)$. Variables such as number of rows and percentage of grain moisture did not show significant differences. It is possible to gradually reduce chemical fertilizers using vermicomposta without affecting grain production.

Keywords: Biomass, Fertility, Maize, Sustainable, Vermicompost.

\section{INTRODUCCIÓN}

Uno de los problemas que más serios que se observan en todo el mundo es la degradación de suelos; en particular la reducción de su fertilidad en zonas agrícolas. Esta, es ocasionada tanto por fenómenos naturales como las lluvias, a través del arrastre y lixiviación de nutrientes, así como de las prácticas de manejo que incluyen el monocultivo, el uso excesivo de agroquímicos y la poca reincorporación de carbono al suelo en forma de materia orgánica, fundamental si se pretende sostener la productividad en los agroecosistemas (Hernández-Rodríguez et al. 2010).

El maíz es uno de los principales cultivos producidos en el mundo y en México su producción ocupa el $50.3 \%$ de la superficie agrícola sembrada, equivalente a 8.07 millones de hectáreas y es el cuarto productor mundial después de Estados Unidos, China y Brasil (SIAP-SAGARPA, 2010).

Michoacán es una de las 7 entidades que concentran el $64.5 \%$ del volumen de producción nacional (22.1 Mt), ocupando en 2012 el tercer lugar después de Sinaloa y Jalisco con $8.2 \%$ del volumen producido (SHCP-FDN, 2014). En Michoacán de las 450 mil hectáreas de maíz sembradas, $81 \%$ corresponde a la agricultura de temporal y $19 \%$ bajo riego. Se cosecharon 1.32 millones de toneladas bajo temporal con rendimiento promedio de 1.7 ton $\mathrm{ha}^{-1}$ y 0.49 millones de toneladas bajo riego con rendimiento promedio de 4.1 ton ha $^{-1}$ (CarreraValtierra, 2013; SHCP-FDN, 2014).

La adición de materiales orgánicos ha recobrado importancia, dada la necesidad de reducir la presencia de compuestos químicos contaminantes en el suelo, agua, aire y productos alimenticios. Dentro de los materiales orgánicos, sobresalen la composta y la vermicomposta, esta última es rica en humus, macro $\mathrm{y}$ micronutrientes (N, Ca, K, Fe, Mn y Zn) además de promover la riqueza microbiológica (Duran y Enríquez, 2007; Hernández-Rodríguez et al. 2010).

Trabajos realizados en cultivo de maíz con aplicación de abonos orgánicos han mostrado buenos resultados como el de López-Martínez et al. (2001) quienes sugirieron una aplicación de 20 a $30 \mathrm{t} \mathrm{ha}^{-1}$ de composta, para la producción de maíz. A nivel de invernadero, Matheus et al. (2007) probó la eficiencia de diferentes materiales orgánicos, solos y combinados 
con fertilizante químico, obteniendo que la combinación de vermicomposta más fertilizante químico favoreció la producción de biomasa seca en el maíz en los primeros 40 días. González et al. (2015) encontró que en campo, una dosis $16 \mathrm{t} \mathrm{ha}^{-1}$ de vermicomposta, iguala el rendimiento de la fertilización convencional.

Michoacán es uno de los estados en los que se tienen sistemas de producción sin rentabilidad privada y se cosechan en estas condiciones aproximadamente 0.684 millones de toneladas de la producción nacional, donde los costos de mano de obra y uso excesivo de fertilizantes explican su falta de rentabilidad (González y Alferes, 2010), además del efecto negativo de los excedentes de agroquímicos sobre la calidad del suelo. Así, la reducción de agroquímicos sin comprometer la productividad del cultivo es una prioridad en nuestro estado, motivo por el cual, se planteó como objetivo del presente trabajo, evaluar en condiciones de campo, el efecto de la aplicación de vermicomposta combinada con dosis de fertilización reducida al $50 \%$, sobre la producción de biomasa vegetativa y de grano, en cultivo de maíz.

\section{MATERIALES Y MÉTODOS}

\section{Área de estudio}

La parcela experimental se ubicó en la comunidad de Joya de la Huerta, municipio de Morelia Michoacán, México. Se localiza en las coordenadas $101^{\circ} 19^{\prime} 14^{\prime \prime}$ y $101^{\circ} 18^{\prime} 17^{\prime \prime}$ de longitud oeste y $19^{\circ} 37^{\prime} 19^{\prime \prime}$ y $19^{\circ} 37^{\prime} 20^{\prime \prime}$ de latitud norte, a una altitud de $2115 \mathrm{~m}$. El clima es del tipo $\mathrm{Cb}(\mathrm{w} 2)(\mathrm{w})\left(\mathrm{i}^{\prime}\right) \mathrm{g}$ : templado subhúmedo con lluvias en verano. Temperatura media anual de $15.6{ }^{\circ} \mathrm{C}$ y precipitación media anual de $820 \mathrm{~mm}$, con oscilación térmica $>5^{\circ} \mathrm{C}$ (Carlón y Mendoza, 2007).
La zona agrícola se encuentra sobre rocas de tipo basáltico y andesítico (Qb) (Ramos, 2012). El suelo corresponde al Luvisol: suelo bien desarrollado con una saturación de bases $\geq 50 \%$ (SEEM-UMSNH, 2003). Textura franco arcillosa, densidad aparente de $1.14 \mathrm{~g} \mathrm{~cm}^{-3}$, porosidad media (50\%), $\mathrm{pH}$ ligeramente ácido (6.3) y contenido medio de materia orgánica $(2.3 \%)$ y una capacidad de intercambio $\geq 25$ $\mathrm{Cmol}(+) \mathrm{kg}^{-1}$, interpretación de acuerdo con Muñoz et al. (2013).

\section{Diseño experimental}

Una parcela de 30 x 30 metros, fue dividida en 36 cuadrantes de $25 \mathrm{~m}^{2}$, con un diseño de bloques al azar y seis repeticiones. Los tratamientos fueron: Tratamiento absoluto (sin fertilizantes) (TA), fertilización química convencional (TC), así como cuatro tratamientos mixtos: vermicomposta a razón de 4, 8, 16 y 32 $\left.\mathrm{t} \mathrm{ha}^{-1}\right)+$ fertilización química convencional (sulfato de amonio/superfosfato triple, relación 3:1), reducida al $50 \%$.

\section{Preparación del terreno y siembra}

En el mes de mayo del 2014 se barbecho con tractor para remover el suelo. Se sembró en la primera semana de junio, con una densidad de 27,200 plantas ha $^{-1}$, equivalentes a 68 plantas/25 $\mathrm{m}^{2}$, con una distancia de $38 \mathrm{~cm}$ entre las plantas y $90 \mathrm{~cm}$ entre los surcos. Como material de siembra se utilizaron semillas de maíz criollo de la región, seleccionados previamente por el agricultor.

\section{Fertilización-abonamiento.}

La aplicación vemicomposta y fertilizantes se realizó a los 40 días después de la siembra. La fertilización convencional consistió en una mezcla de 3 bultos de sulfato de amonio (20.500-00), con un bulto de súper fosfato triple de calcio (00-46-00). De ésta mezcla se aplicó 1.6 $\mathrm{kg}$ por tratamiento equivalente a 22.22 gramos por planta. Las cuatro diferentes dosis de 
vermicomposta se aplicaron alrededor de las plantas, adicionando $800 \mathrm{~g} / 25 \mathrm{~m}^{2}$ de fertilizante convencional como auxiliar para el crecimiento de las plantas, que equivale a 11.11 gramos para cada planta, es decir, el 50\% de lo que se aplica normalmente.

\section{Desarrollo vegetativo}

Durante el desarrollo de las plantas, se realizaron 1 a 2 visitas por mes, para tomar los datos de tamaño y grosor de las plantas, iniciando en junio cuando estas tuvieron un tamaño 20-25 $\mathrm{cm}$, hasta la etapa cosecha (noviembre), a razón de 20 plantas por cuadrante (120 por tratamiento).

La altura se midió con una cinta métrica desde el nivel del suelo a la base de la espiga y el grosor del tallo se midió aproximadamente a $30 \mathrm{~cm}$ del suelo, con un vernier.

\section{Obtención de material vegetal.}

El corte de las plantas se realizó a los 4 meses y medio después de la siembra del maíz, cuando de detuvo el crecimiento vegetativo. En cada cuadrante se cortaron 3 plantas para determinar la biomasa de cada una de sus partes: las hojas y mazorca se separaron manualmente en tanto el tallo se cortó a ras del suelo y se fragmento para depositarse en bolsas de papel estraza. La extracción de las raíces se llevó a cabo con la ayuda de una pala, eliminando en lo posible el suelo adherido a las mismas y depositadas en bolsas de plástico.

El material vegetal obtenido en campo: hojas, tallos, mazorcas y raíces, se transportó en bolsas de papel ó de plástico (raíces) al laboratorio de Edafología de la Universidad Michoacana.

\section{Trabajo de laboratorio.}

\section{Determinación de Biomasa.}

Las diferentes partes de las plantas (hojas, tallo, mazorcas) fueron colocadas en una secadora botánica a $60 \pm 1^{\circ} \mathrm{C}$, hasta obtener peso constante.
Las raíces fueron lavadas cuidadosamente para eliminar el suelo adherido a las mismas, utilizando malla de $1 \mathrm{~mm}$, para evitar en lo posible pérdida de raíces finas. Una vez limpias se colocaron en bolsas de papel estraza para su secado.

\section{Variables evaluadas del fruto (mazorca).}

Se obtuvo el peso de la mazorca, que considera el peso total (granos + olote), el peso del grano, utilizando una balanza semi-analítica (0.01 de precisión) marca Sartorius Modelo PT600, además de la longitud, diámetro, número de hileras y porcentaje de humedad del grano. Considerando la biomasa de grano y el número de plantas por superficie, se calculó el rendimiento en ton $\mathrm{ha}^{-1}$.

\section{Análisis estadísticos.}

Se realizó un análisis de varianza y comparación de medias de Tukey $(\mathrm{p}<0.05)$ para los parámetros que se evaluaron como la biomasa de las plantas y las diferentes variables de la mazorca, utilizando el programa JMP v.6.0.0 (SAS Institute Inc. 2005).

\section{RESULTADOS}

\section{Desarrollo vegetativo}

Los datos registrados mostraron que el crecimiento acelerado del maíz para obtener su talla máxima fue de 3 meses, de junio a agosto. A partir del mes de septiembre el crecimiento fue mínimo y permaneció prácticamente constante (Fig. 1). Se aprecia que los tratamientos V32, V16, V8 y TC, favorecieron el tamaño de las plantas, quedando claramente el testigo absoluto (TA) con la talla más baja y después el tratamiento V4.

En cuanto al grosor del tallo, este se alcanzó más rápidamente, con un máximo en la primera semana de agosto (Fig. 2). 


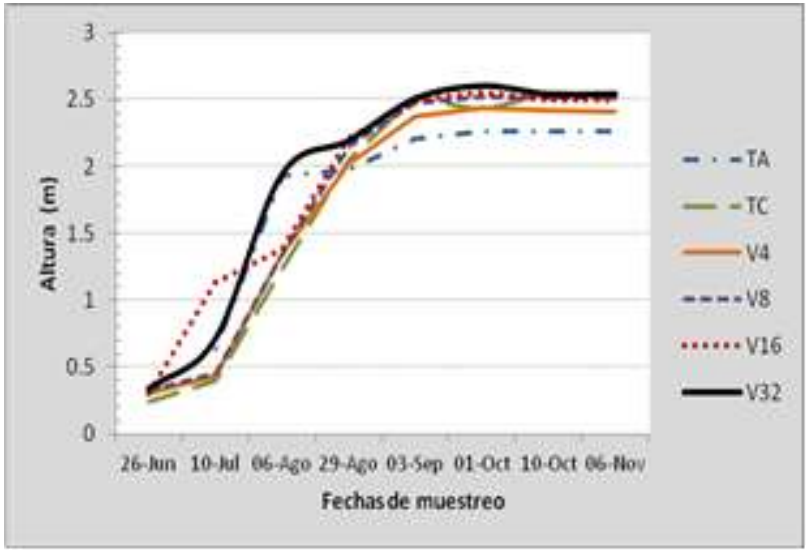

Figura 1. Curva de crecimiento del cultivo.

\section{Producción de biomasa}

Hojas. En la producción de biomasa hojas, únicamente el tratamiento V32 mostró diferencias significativas respecto al control. No

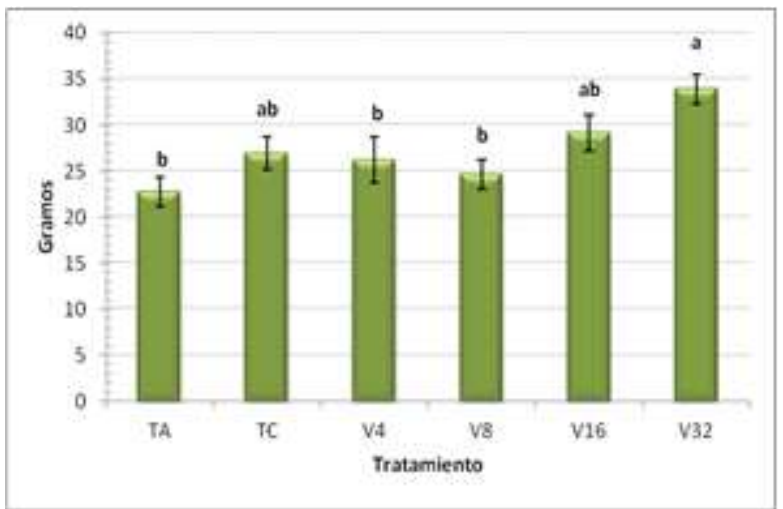

Figura 3. Comparación en la producción de biomasa de hojas. Tukey $(\alpha \leq 0.05)$.

Tallos: La producción de biomasa en tallos mostro diferencias significativas de los tratamientos con vermicomposta V16 y V32, respecto al control absoluto (Fig. 4).

En los dos casos anteriores, el tratamiento convencional (TC) no mostro diferencias significativas respecto al control (TA).

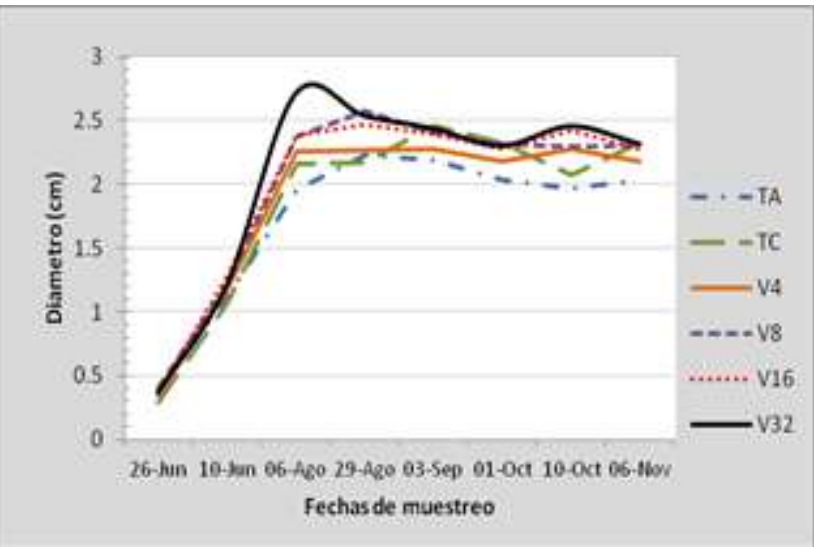

Figura 2. Curva de engrosamiento del tallo.

obstante se observa claramente una tendencia a incrementarse la biomasa al aplicarse mayor dosis de vermicomposta (Fig. 3).

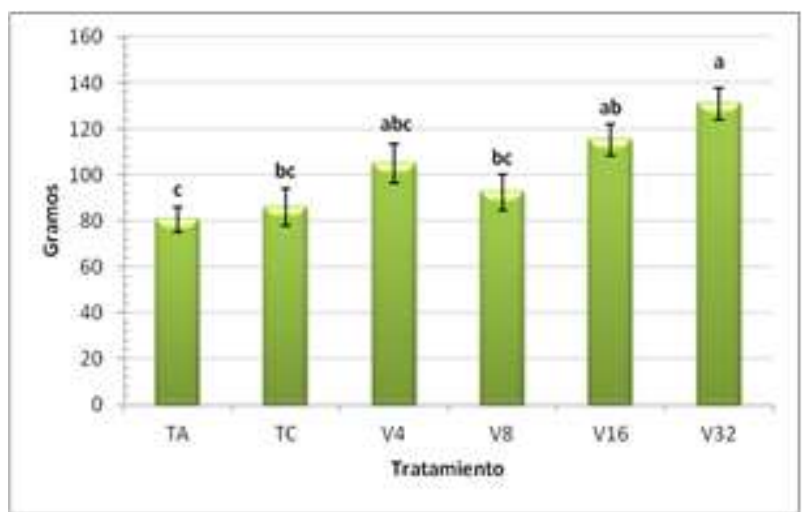

Figura 4. Comparación en la producción de biomasa de tallos. Tukey $(\alpha \leq 0.05)$.

Raíz: La producción de biomasa de raíz, mostró una clara tendencia a incrementarse al aumentar la dosis de vermicomposta, no obstante, solo el tratamiento V32, mostró diferencia significativa respecto al control absoluto como se muestra en la Fig. 5. 


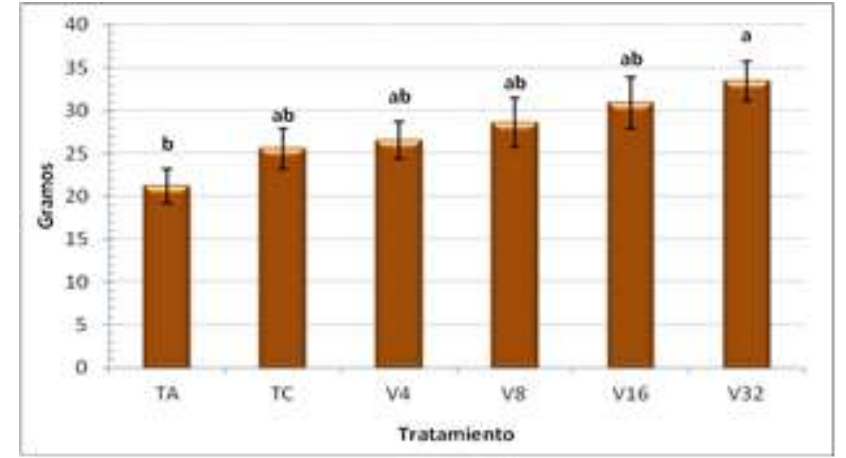

Figura 5. Comparación en la producción de biomasa de raíz. Tukey $(\alpha \leq 0.05)$.

Mazorca: La producción de biomasa de mazorca, mostro diferencias significativas de los tratamientos V8, V16 Y V32, respecto al control absoluto, compitiendo con estos el TC.

A continuación se muestran los resultados de las variables asociadas a la productividad: biomasa de grano, longitud, diámetro, número de granos y número de hileras por mazorca.

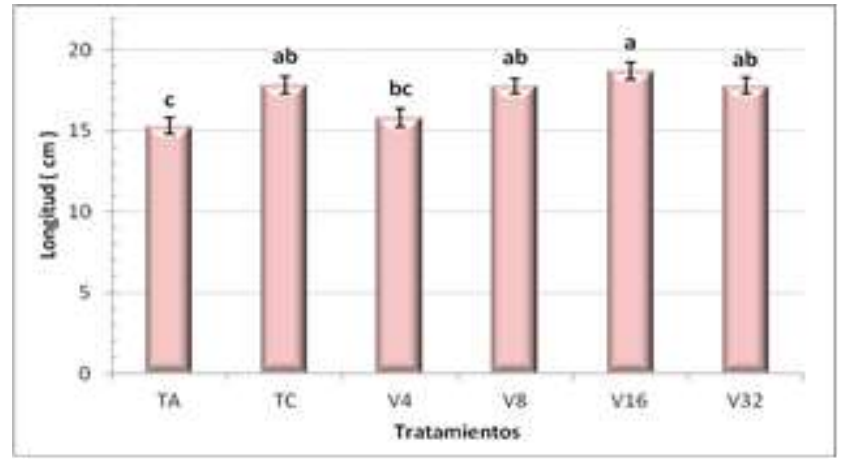

Figura 7. Comparación en la longitud de la mazorca. Tukey $(\alpha \leq 0.05)$.

Longitud de la mazorca: En la longitud de la mazorca, se observó diferencia significativa del tratamiento V16 respecto TA y V4, pero no se diferenció del resto de los tratamientos (Fig. 7).

Diámetro de la mazorca: El diámetro de la mazorca mostro diferencia significativa del tratamiento V32, respecto al control absoluto, pero no se diferencia de los tratamientos V8, V16 y TC (Fig. 8).

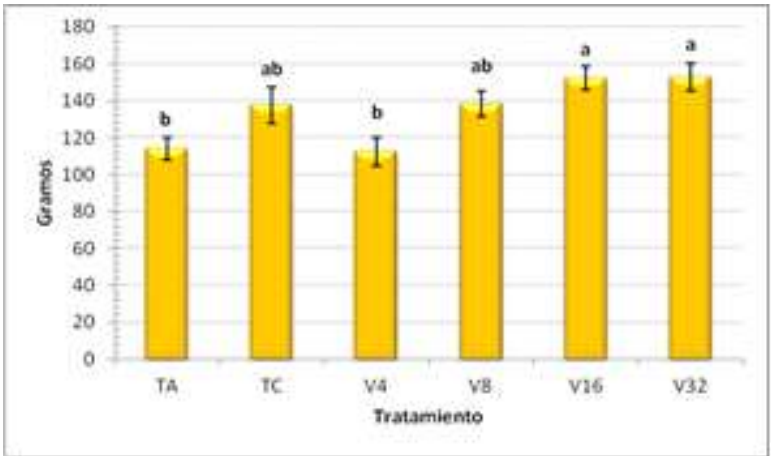

Figura 6. Comparación en la producción de biomasa de grano. Tukey $(\alpha \leq 0.05)$.

Granos de maíz: La producción de biomasa del grano, presento un comportamiento similar a lo obtenido para la mazorca completa, lo cual da consistencia a los resultados obtenidos. Los tratamientos V16 y V32, mostraron diferencias significativas respecto al control absoluto (Fig. 6), lo cual repercute en la producción de maíz, en términos de toneladas por hectárea (Tabla 1).

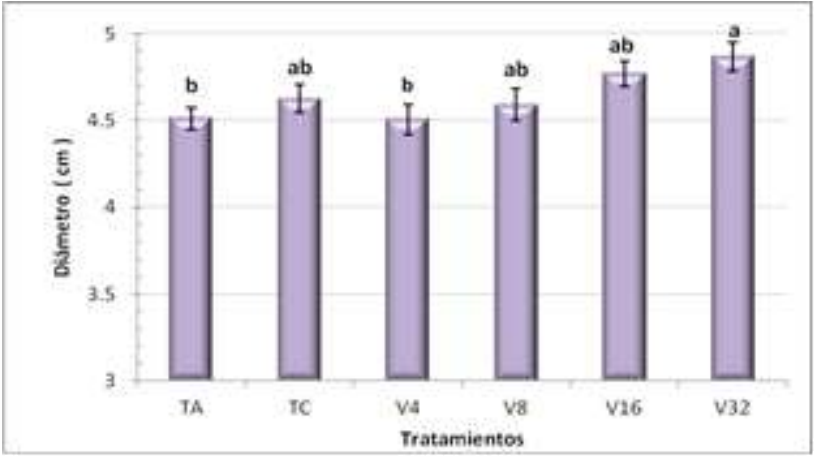

Figura 8. Comparación del diámetro de la mazorca. Tukey $(\alpha \leq 0.05)$.

Las variables número de granos por mazorca, número de hileras por mazorca y humedad del grano, no mostraron diferencias significativas entre tratamientos, como se muestra en la Tabla 1. 
Tabla 1. Otras variables asociadas a la productividad.

\begin{tabular}{|c|c|c|c|c|c|c|c|c|}
\hline & \multicolumn{2}{|c|}{ Rendimiento t ha ${ }^{-1}$} & \multicolumn{2}{|c|}{ Número granos } & \multicolumn{2}{|c|}{ Número hileras } & \multicolumn{2}{|c|}{ \% humedad } \\
\hline Tratamiento & $\mathrm{Me}$ & & & & $\mathrm{Me}$ & & & \\
\hline TA & 2.386 & $\mathrm{~b}$ & 330 & $\mathrm{a}$ & 12.66 & $\mathrm{a}$ & 7.19 & $\mathrm{a}$ \\
\hline $\mathrm{TC}$ & 3.171 & $a b$ & 383 & $\mathrm{a}$ & 12.55 & $\mathrm{a}$ & 7.15 & $\mathrm{a}$ \\
\hline V4 & 2.882 & $a b$ & 316 & $\mathrm{a}$ & 12.05 & $\mathrm{a}$ & 7.33 & $\mathrm{a}$ \\
\hline V8 & 4.156 & $\mathrm{a}$ & 376 & $\mathrm{a}$ & 12.44 & $\mathrm{a}$ & 7.25 & $\mathrm{a}$ \\
\hline V16 & 4.552 & $\mathrm{a}$ & 389 & $\mathrm{a}$ & 12.66 & $\mathrm{a}$ & 6.95 & $\mathrm{a}$ \\
\hline V32 & 4.466 & $\mathrm{a}$ & 380 & a & 12.77 & $\mathrm{a}$ & 7.40 & $\mathrm{a}$ \\
\hline
\end{tabular}

Medias con la misma letra en la misma columna no son significativamente diferentes, Tukey $(\alpha \leq 0.05)$.

\section{DISCUSION}

Los resultados obtenidos mostraron que la producción de biomasa seca estuvo asociada a las diferentes dosis de vermicomposta, obteniendo las medias más altas en los tratamientos V16 y V32. Lo anterior concuerda con lo reportado por López-Martínez et al. (2001) quien comparó la eficiencia de diferentes abonos orgánicos y un testigo químico en la producción de maíz, encontrando que la composta fue eficiente a dosis de 20 a $30 \mathrm{t} \mathrm{ha}^{-1}$. Los resultados obtenidos mostraron que si existe un efecto residual de la vermicomposta aun combinada con el fertilizante químico, lo cual no fue encontrado por Matheus et al. (2007), pero se atribuye a las condiciones y dosis aplicadas de los materiales. Asimismo González et al. (2015) quien utilizo una aplicación máxima de $16 \mathrm{t} \mathrm{ha}^{-1}$ de vermicomposta reporto valores similares en la producción de grano para la fertilización química y orgánica. Duran y Enríquez (2007) sugieren que el efecto individual de la vermicomposta es gradual y no se refleja de inmediato, siendo necesaria la aplicación por periodos largos de tiempo. Para este estudio, consideramos que la aplicación combinada de fertilizante químico más la aplicación de vermicomposta produjo un efecto sinérgico que potencializo el efecto sobre la producción de biomasa vegetal y de grano. Así, retomar el uso de los abonos orgánicos en forma gradual parece ser una buena estrategia para reducir el uso excesivo de agroquímicos (Turrent et al. 2012), mejorar el suelo y favorecer el medio ambiente.

\section{CONCLUSIONES}

El desarrollo y producción de biomasa en raíz, tallo, hojas y fruto está asociado a las dosis de vermicomposta aplicada. La aplicación de 16 o $32 \mathrm{t} \mathrm{ha}^{-1} \mathrm{y}$ dosis reducida de fertilización química al 50\% mantiene la producción de grano y biomasa vegetativa, beneficiando el rendimiento y producción de forraje para alimentación de ganado.

\section{LITERATURA CITADA}

Carlón A.T. y M.E. Mendoza. 2007. Análisis hidrometeorológico de las estaciones de la cuenca del lago de Cuitzeo. Investigaciones Geográficas, Boletín del Instituto de Geografía, UNAM, (63): 56-76

Carrera Valtierra, J. A. 2013. Estudio de la diversidad genética y su distribución de los maíces criollos y sus parientes silvestres en Michoacán. Universidad Autónoma de Chapingo. Centro Regional Universitario 
Centro Oriente. Informe final SNIBCONABIO. Proyecto No. FZ001. México, D.F.

Duran L. y Enriquez C. 2007. Caracterización química, física y microbiológica de vermicompostes producidos a partir de cinco sustratos orgánicos. Agronomía Costarricense 31(1): 41-51

González, E. A. y Alferes, V.M. 2010. Competitividad y ventajas comparativas de la producción de maíz en México. Revista Mexicana de Ciencias Agrícolas. 1(13): 381396

González, R,F., González, C.J.C., Alcalá de J. M. y Ramírez, M.C.A. 2015. Producción sostenida de maíz, utilizando fertilización mixta en agroecosistemas de temporal. Ciencia Nicolaita, (65), 139-164.

Hernández-Rodríguez, O. A., Ojeda-Barrios, D. L., López-Díaz, J. C., \& Arras-Vota, A. M. 2010. Abonos orgánicos y su efecto en las propiedades físicas, químicas y biológicas del suelo. Tecnociencia, Chihahua. 4(1): 1-6 López-Martínez J.D., A. Díaz E., E. Martínez R. y R. Valdez C. 2001. Abonos orgánicos y su efecto en propiedades físicas y químicas del suelo y rendimiento en maíz. Terra 19(4): 293-299

Matheus L., J. Caracas., F. Montilla y O. Fernández.
2007. Eficiencia agronómica relativa de tres abonos orgánicos (vermicompost, compost, y gallinaza) en plantas de maíz (Zea mays). Agricultura Andina, 13: 27-38

Muñoz, I. D.J, Soler A. A., López G. F. y Hernández M.M.M. 2013. Edafología, manual de métodos de análisis de suelo. UNAM, Iztacal, México. 139 pp.

SIAP-SAGARPA， 2010. Situación actual y perspectivas del maíz en México 1996-2010. 174 pp.

SHCP-FND, 2014. Panorama del maíz. http://www.financierarural.gob.mx/informa cionsectorrural/Panoramas/Ficha\% $20 \mathrm{Ma} \%$ C3\%ADz.pdf (Consultada: 15 abril de 2017).

SEEM-UMSNH. 2003. Atlas Geográfico del Estado de Michoacán. $2^{\text {a }}$ Edición, EDDISA S.A. de C.V., México. 308 pp

Turrent, F. A., T.A. Wise y E. Garvey. 2012. Factibilidad de alcanzar el potencial productivo de maíz de México. $36 \mathrm{pp}$. http://www.ase.tufts.edu/gdae/Pubs/wp/1203TurrentMexMaizeSpan.pdf (Consultada: 09 Mayo de 2017). 


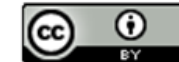

Este tex to está protegido por una licencia licencia Creative Commons 4.0

Usted es libre para Compartir —copiar y redistribuir el material en cualquier medio o form ato- y Adaptar el documento $\rightarrow$ remezclar, transformar y crear a partir del material- para cualquier propósito, , incluso para fines comerciales, siempre que cumpla la condición de:

Atribución: Usted debe dar crédito a la obra original de manera adecuada, proporcionar un enlace a la licencia, e in dicar si se han realizado cam bios. Puede hacerlo en cualquier form a razonable, pero no de form a tal que sugiera que tiene el apoyo del licenciante o lo recibe por el uso que hace de la obra.

Resumendelicencia - Textocompletodelalicencia 